\title{
TOWARDS DEVELOPMENT OF ROBOTIC AID FOR REHABILITATION OF LOCOMOTION-IMPAIRED SUBJECTS
}

\author{
Dr. Antal K. Bejczy \\ Senior Research Scientist \\ Jet Propulsion Laboratory \\ California Institute of Technology \\ Pasadena, California 91109 \\ Phone: (818) 354-4568,Fax: (818) 393-5007,e-mail: akbejczy@jpl.nasa.gov
}

\begin{abstract}
Manual assistance of therapists to help movement of legs of spinal cord injured (SCI) subjects during stepping on a treadmill for locomotion rehabilitation has severe economic and technical limitations. Scientists at the Department of Physiological Science at the University of California Los Angeles (UCLA) and roboticists at the Jet Propulsion Laboratory (JPL) initiated a joint effort to develop a robotic mechanism capable of performing controlled motions equivalent to the arm and hand motions of therapists assisting the stepping of locomotion impaired subjects on a treadmill, while the subjects' body weight is partially supported by an overhead harness. A first necessary technical step towards this development is to measure and understand the kinematics and dynamics of the therapists' arm and hand motions as they are reflected on the subjects' leg movement. This paper describes an initial measurement system developed for this purpose together with the related measurement results, and outlines the planned future technical work.
\end{abstract}

Key Words: Biped Locomotion, Rehabilitation, Robotic Aid, Force-Torque and Acceleration Sensors.

\section{INTRODUCTION}

Each year more than a half million people are hospitalized in the USA due to head and central nervous system injuries and diseases. Most of them are spinal cord injury and stroke patients. These injuries result in partial or total paralysis. Some of them will suffer life-long physical impairment, but about $70 \%$ of these patients could be retrained to walk since medical experiments show that the lower spinal cord, without input from the brain, can learn specific motor lasks that are consistently practiced. For instance, it can learn to step under full weight-loading conditions over a range of speeds, and stand. These experiments suggest that a significant degree of functional neural regeneration can be achieved by proper practice or training. (More on the related medical research background can be found in Refs. 1-3).

Current technique for rehabilitation of stepping skills is based on an approach called Body Weight Suspended Training (BWST) on a treadmill. In BWST rehabilitation exercises, therapists manually assist the movement of the legs of spinal cord injured (SCI) subjects during stepping on a treadmill while the subject's body weight is partially supported by an overhead harness. The therapists move the legs of the subject following the treadmill speed and exert pressure on the leg in all directions 10 maintain as normal walking motion as physically possible.

Note that this type of training of paraplegic subjects requires two therapists, one for each leg. 
since moving one leg over a treadmill and exerting pressure on that leg in all directions requires both hands of a therapist. This fact is the first inherent limitation of the current BWST technique since the considerable human resources needed for using this technique can only be provided to very few subjects today. The second inherent limitation of the current BWST technique is the fact that the movements of the manually manipulated limbs only approximate, but do not replicate, the normal kinematics and dynamics of the human legs during stepping.

The above considerations motivated scientists at the Department of Physiological Science at UCLA and roboticists at JPL to search for a robotic aid solution for rehabilitation of locomotion-impaired subjects. It is assumed that a proper robotic aid would decrease the use of human resources during BWST exercises and, at the same time, increase the quality of training by moving the entire leg in a normal kinematic pattern for a range of locomotion speeds.

The first necessary technical step towards the development of a suitable robotic aid machine is to measure and understand the kinematics and dynamics of the therapists' arm and hand motions as they are reflected on the subjects' leg movements on the treadmill during BWST exercises.

\section{MEASUREMENT OF THERAPIST'S HAND ASSIST ANCE}

We devcloped a two-hand force-torque (F/T) measurement system with the objective in mind to enable a quantitative analysis of the dynamic interaction between a therapist's hands and a locomotion-impaired patient's leg during rehabilitatise locomotion training of a patient on a BW'ST treadmill sel-up at UC'L.A.
The overall two-hand measurement system is shown in Figure 1. Figure 2 shows the placement of the two sensors on a patient's leg. Note that the padded mechanical element is firmly attached to the patient's leg at the indicated two places. Note also that the mechanical elements fitted to the therapist's right and left hands permit a convenient firm grasp/hold of the measurement system without direct touch or contact of the patient's leg. This assures that all forces and torques between the therapist's hands and the patient's leg will go through the sensors during locomotion retraining of the patient.

Each sensor is a six-dimensional vector sensor, measuring the three orthogonal forces and three orthogonal torques at the point of interaction between the therapist's hand and the patient's leg. The frame of reference of the sensors is a Cartesian frame as indicated in Figure 2. The same type of sensor system was developed for and used in teleoperator and robotics research work at the Jet Propulsion Laboratory (JPL) in the late 1970 's - early 1980's. A large version of the sensor was space-tested on the Space Shuttle robot arm in 1994. The sensor system applied in this rehabilitation research is a commercialized version of the original JPL sensor system transferred by JPL to industry in the mid 1980's. (See Ref. 4.)

The physical dimensions of the sensor applied in this rehabilitation research is shown in Figure 3 in millimeters. The sensor is called "mini-sensor" because of its dimensions and dynamic range. The basic mechanical structure of the sensor is of the Maltese Cross configuration as shown in Figure 4 which also shows the basic output frame of the full sensor and of each beam of the Maltese Cross. The actual transducer elements are silicon-based semiconductor strain gages bonded to the four deflection bars of the Maltese Cross. As indicaled on Figure 4 , the full sensor provides cight output readings $(w 1, \ldots \ldots \ldots \ldots, w \&)$ from the strain gages through cight clectric bridge arrangements. 
The eight output readings are then converted to three orthogonal force and three orthogonal torque values through a 6 by 8 transformation matrix.

The dynamic range of the sensor applied in this rehabilitation research work is the following: $F x$ and Fy: $10 \pm \mathrm{lb}$, with resolution of $0.15 \mathrm{oz}, \mathrm{Fz}: 30$ $\pm \mathrm{lb}$, with resolution of $0.45 \mathrm{oz}$, and Tx,Ty,Tz: 20 \pm in-lb, with resolution of 0.15 in-lb. The small transducer bodies are of extremely strong stainless steel which, for the indicated dynamic range, does not require extra overload protection.

The amplifying and multiplexing electronics is located about 2 meters from the sensor head and provide $1 \mathrm{KHz}$ digital output readings. (This digital reading for the UCLA application has been reconverted to analog data since the input end of the UCLA data recording system is analog.) In essence, 1000 readings are available for each second of dynamic interaction between the therapist's hands and the patient's leg. This provides an extremely good data base for analysis.

The functionality of the F/T sensor system was first tested on a "simulated patient" assisted by a therapist at the knee. The measured results are shown in Figure 5 which should be read from the right to the left to see the "simulated improvements" in the patient's walking condition on the BWST treadmill through the recorded F/T assistance effort of the therapist. The comparison of the curves from the right to the left is selfexplanatory for the improved walking condition of the "simulated patient".

\section{MEASUREMENT RESULTS}

Several measurement cycles were carried out on SCI patients at UCLA to evaluate the utility of the installed two-hand F/T sensor system. The results were very encouraging. Here we only quote a part of a case study as an illustration.
Figure 6 shows an actual case geometry, and Figure 7 shows a 10 -sccond scgment of related graphically recorded data. First. a few notes are in place here about Figure 7. (i) The numbers on the horizontal axis denote seconds, while the numbers on the vertical axis denote lbs for force, $F$, and inlbs for torque,T. (ii) The 10-second stepping segment in Fig. 7 starts at the 10th second, because in the previous 10 seconds the therapist was mostly occupied by placing properly the subject's leg over the treadmill; but after the 20th second, several continuing 10-second segments were also recorded, displaying very nearly the same F/T assistance pattern as shown in Fig.7. (iii) Only 8 parallel data sets could be printed out on the available equipment at UCLA. We selected the 8 most important ones out of the measured 12 variables: 3 knee and 3 ankle forces and 2 knee torques.

Before analyzing the data shown in Fig.7, it is necessary to point out that the $F / T$ data measured by the two-hand sensor system only depict the effort of the therapist as seen by the patient's leg motion on the treadmill, which is the real purpose and utility of the F/T sensor system. But this sensor system can not measure the total torques acting at the subject's hip, knee and ankle joints, since the total torque is a vector sum of three torque vectors. Symbolically,

$T($ total $)=T($ voluntary $)+T($ treadmill $)+T($ therapist $)$

where $T$ (voluntary) denotes the amount of torque that the patient can deliver (hopefully on an increasing level after more training), and $\mathrm{T}$ (treadmill) denotes the torque originating from the patients contact with the treadmill. A reasonable estimate, however, can be derived from Fig. 7 for torque values acting at the subject's hip. knee and ankle joints originating from the therapist's assistance, as follows.

In Figure 7 ino full step cycles are marked up secpuentially between 11.9 - 1.3.9) and 1.3.9 - 15.9) 
seconds time segments. (Also indicated there are the "forward" and "backward" states of leg motion within each cycle.) Assuming the orientation of the two $F / T$ sensor axes as shown in Figure 6 that is: $F x(k n e e)$ is parallel to $F z(a n k l e), F z(k n e e)$ is parallel to Fx(ankle), and Fy(knee)is parallel to Fy(ankle) - and assuming that their relative orientation during leg motion remains constant, then we can write the following force components as additive elements (of course, by taking account of their \pm signs):

$\mathrm{Fx}($ knee $)+\mathrm{Fz}$ (ankle) $=\mathrm{F}$ (up-down)

$\mathrm{Fz}($ knee $)+\mathrm{Fx}$ (ankle) $=\mathrm{F}$ (forward-backward $)$

$F y(k n e e)+F y($ ankle $)=F($ sideways $)$

where "up-down," "forward-backward" and "sideways" denote orthogonal orientations relative to the treadmill belt or, in other words, relative to the vertical plane of the leg motion.

Based on the approximate equalities (1), (2) and (3), the following approximate average force values hold during the first step cycle (that is, in the $11.9-13.9$ seconds time segment):

$\begin{array}{lll}\text { F(up/forward) } & 1.8 & \text { lbs } \\ \text { F(forward) } & 9.0 & \text { lbs } \\ \text { F(sideways/forward) } & 1.0 & \text { lbs } \\ \text { F(down/backward) } & 8.0 & \text { lbs } \\ \text { F(backward) } & 8.0 & \text { lbs } \\ \text { F(sideways/backward) } & 1.0 & \text { lbs }\end{array}$

From the above force values one may estimate about 30.97 in-lbs backward/forward (pitch) torque at the hip joint. The quite variable and sometimes quite high roll torque, $\mathrm{Tx}(\mathrm{knee})$, acting at the hip joint has a maximum value up to 28 inlbs.

The pitch torque acting at the knee joint can be assumed to be represented by Ty(knee) only, with an average value of 20 in-lhs during backward motion. - The average 1.0 lbs $\Gamma x($ ankle) force together with the 8.0 ths (down/backward) foree that secmingly could mitigate the measured value of the Ty(knee) during backward motion actually serve to help keep the foot in contact with the moving belt. Therefore, they have no direct influence on the knee pitch torque, only indirect through the moving belt.

It is noted that the numerical values quoted above carry about $\pm(20-25) \%$ uncertainty or possible error because of the various assumptions used in their derivation. In particular, a more accurate estimate would require to calculate the relevant Jacobian matrices. That was omitted here because of the incomplete nature of the available data sets.

\section{CONCLUSIONS AND FUTURE WORK}

The available $\mathrm{F} / \mathrm{T}$ data provide very reasonable bounds for the dynamic range of the therapist's interaction with the treated subject's leg on a BWST treadmill system. The data should be refined, however, in two directions in order to gain a better quantitative understanding of the treadmill's impact on relearning the biped locomotion by the patient. The first direction will imply the modeling of the cumulative force and torque generated by the treadmill on the patient's leg. The second direction will imply the measurement of the kinematic error signature generated by the subject's leg behavior during the training process, together with the therapist's control response to that error signature.

The kinematic error signature can be best captured by measuring the acceleration interaction between the subject's leg and the therapist's hand. A key element in the future work is, therefore, to augment the $F / T$ sensor measurements with accelerometer measurements. The installation of a combined Force-Torque-Acceleration (F/T\&A) sensor system is in progress. The sensor is commercially arailable, see Ref. 5 . 
The ultimate goal of this specific clement of a broader UCLA-JPL Neurolab project is to design and develop a robotically controlled prototype mechanical system, attached to the treadmill frame, capable of performing mechanical actions equivalent to the arm and hand actions of a therapist who is assisting the stepping of a locomotion-impaired subject on a treadmill, while the subject's body weight is partially supported by an adjustable overhead harness. It is envisioned, that the robotic mechanism will have suitable elements (called "links") which can be connected to the legs of a subject at locations distal to the petexlla to assist with knee motions, and distal to the ankle to assist with foot swing and placement as necessary. The "links" will be equipped with suitable sensors near the links' contact connection to the legs, that is, near to the links' "output end." It is also envisioned that the development of the related sensing-based robotic control intelligence will be a very challenging but rewarding future work.

\section{ACKNOWLEDGMENTS}

The research in this paper was carried out at the Jet Propulsion Laboratory, California Institute of Technology, under contract with the National Aeronautics and Space Administration. The contribution of Drs. B. Jau and P. Fiorini of JPL to this work is gratefully acknowledged. The training measurements and data recording at UCLA were carried out by Drs. S. Harkema and Uday Patel. The work was supported by Professors B.H. Dobkin and V.R. Edgerton of UCLA and by Program Manager J. Weiss of JPL.

\section{REFERENCES}

[1] Dobkin, B.H., New frontiers in spinal cord injury rehabilitation. J Neurologic Rehabilitation 8:33-39, 1994

[2] Edgerton, V.R., Roy, R.R., Hodgson, J.A., Prober, R.J., de Guzman, C.P. and de Leon, R. A physiological basis for the development of rehabilitative strategies for spinally injured patients. J. Am. Paraplegia Soc. 14:150-157, 1991.

[3] Harkema, S.J., P.S. Requejo, S.L. Hurley, B. Mares, B.H. Dobkin, and V.R. Edgerton. Load and phase dependent modulation of motor pool output by the human lumbar spinal cord during manually assisted stepping. Neurons, Networks, and Motor Behavior. 1:69, 1995.

[4] Current F/T Sensors is from ATI Industrial Automation, Garner, NC 27529, USA - Fax: 919-792-8259.

[5] Combined F/T\&A sensors is from JR3, Inc., Woodland, CA 96776, USA - Fax: 530-6613701. 


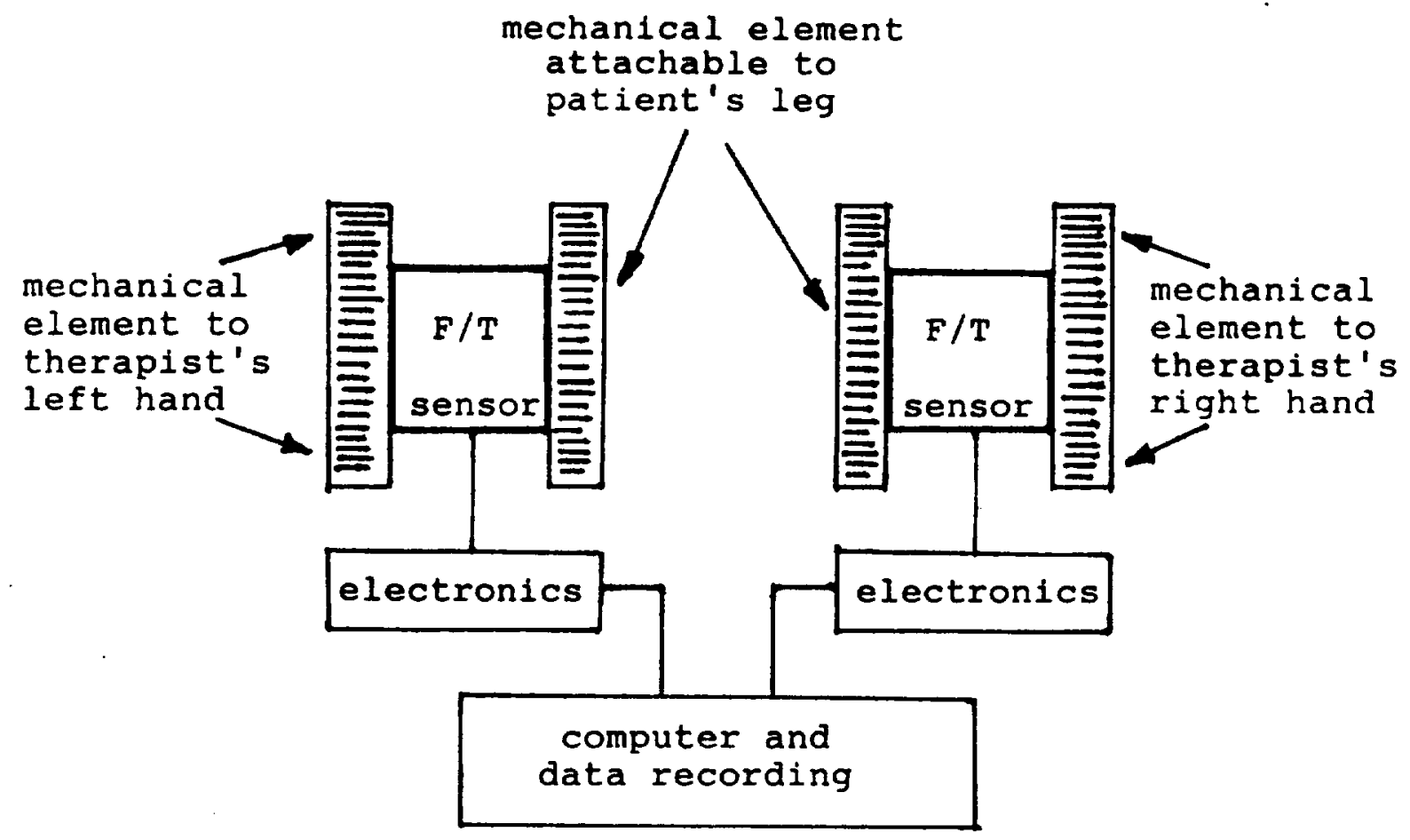

Figure 1. Two-hand Force-Torque (F/T) Measurement System

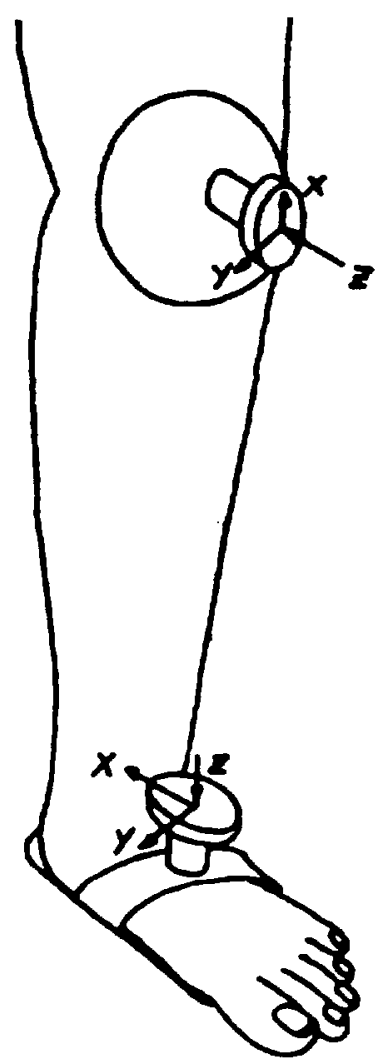

Figure 2. Placement of $\mathrm{F}, \mathrm{T}$ Sensors

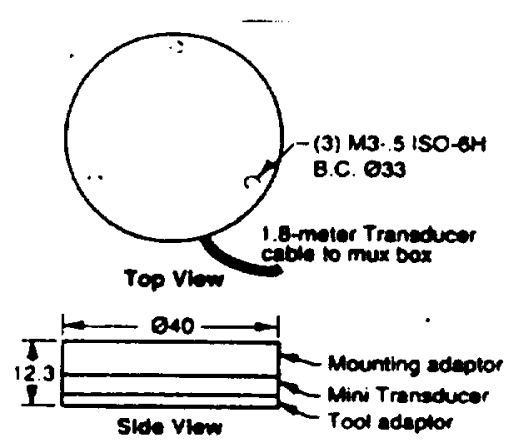

Figure 3. Dimensions of Mini F/T Sensors (in mm)

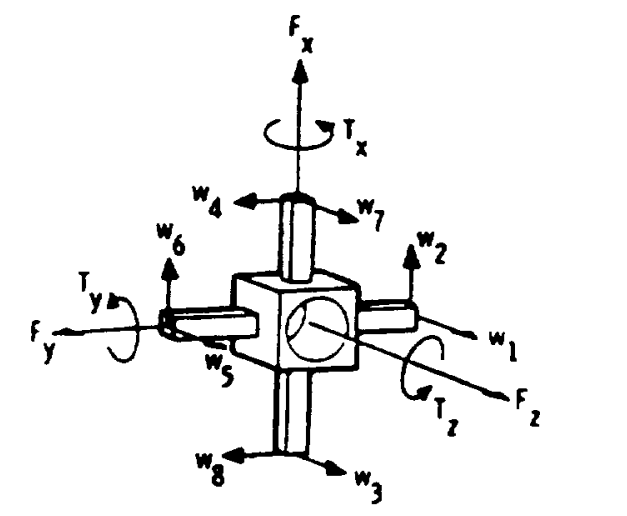

Figure 4. F,T Sensor Mechanical Frame with Output in Cartesian rrame 
Forces exerted by the trainer while the subject stepped with varying levels of effor

Full eflort by subject

Half offon by subject

No eflon by subpect
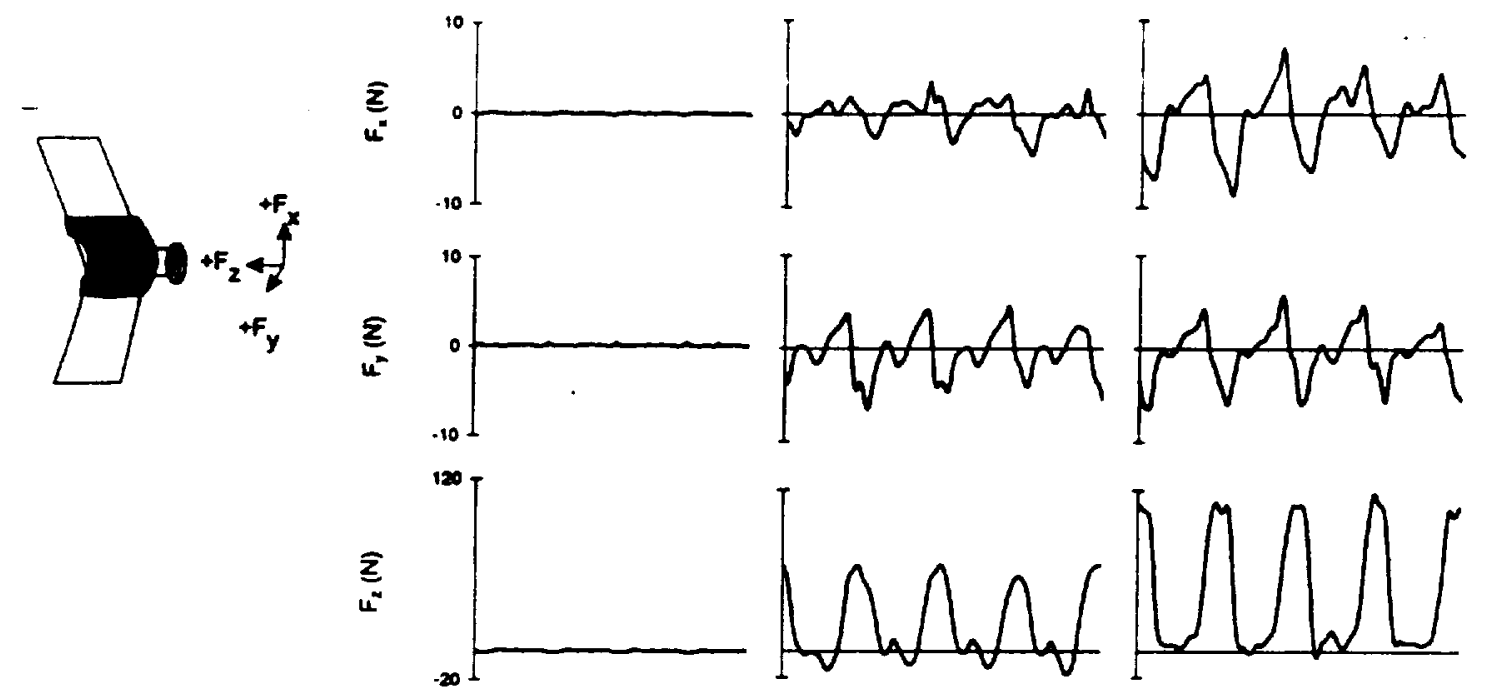

Figure 5. F/T Sensor Functionality Test at Knee

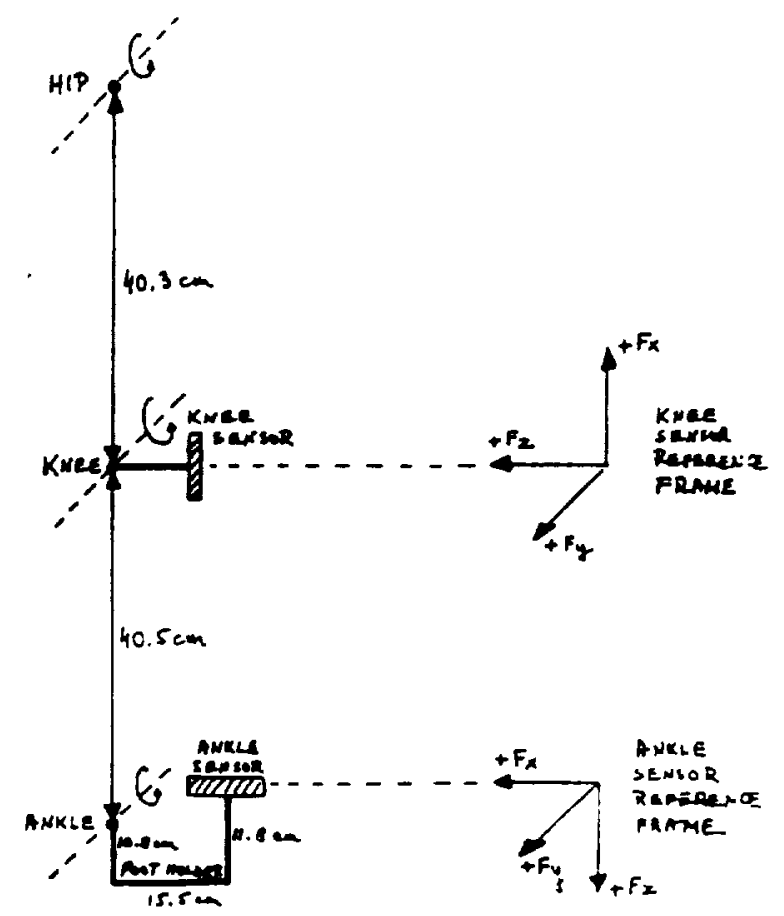

Figure 6 . An Application Case (iemelr 
PX DIRE

Py pae

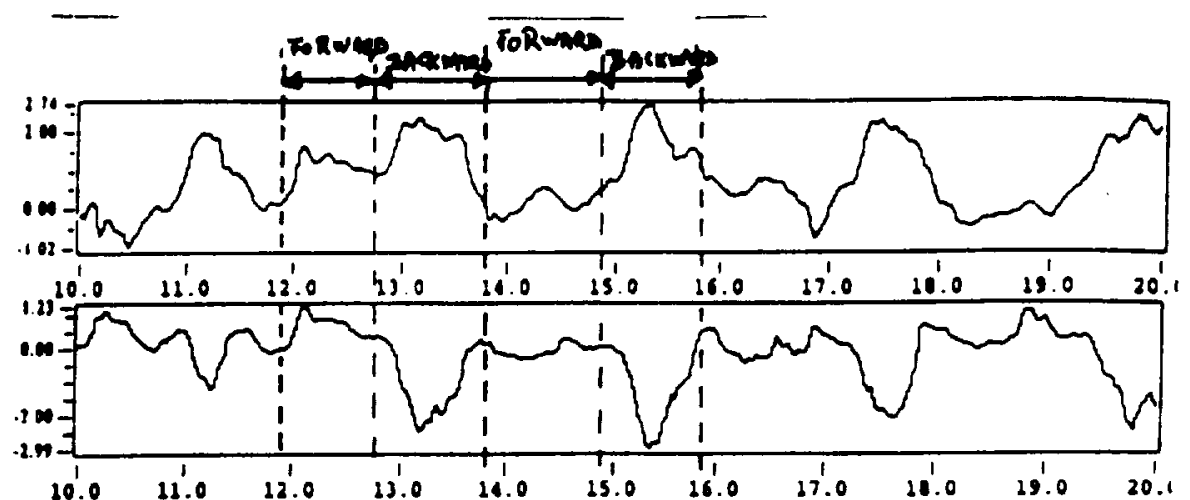

r. nis

$\boldsymbol{\Gamma}$ Mure

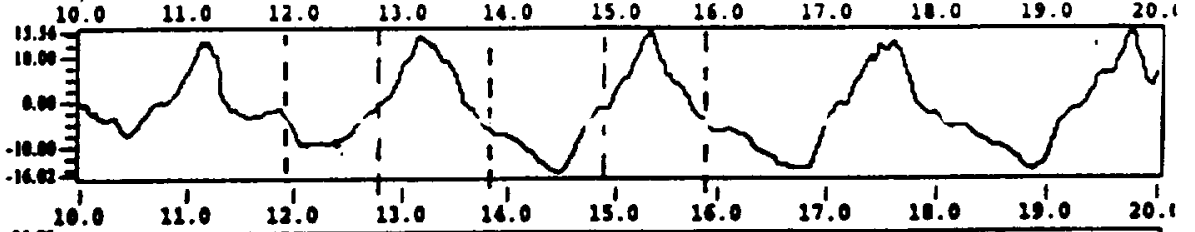

II DA.
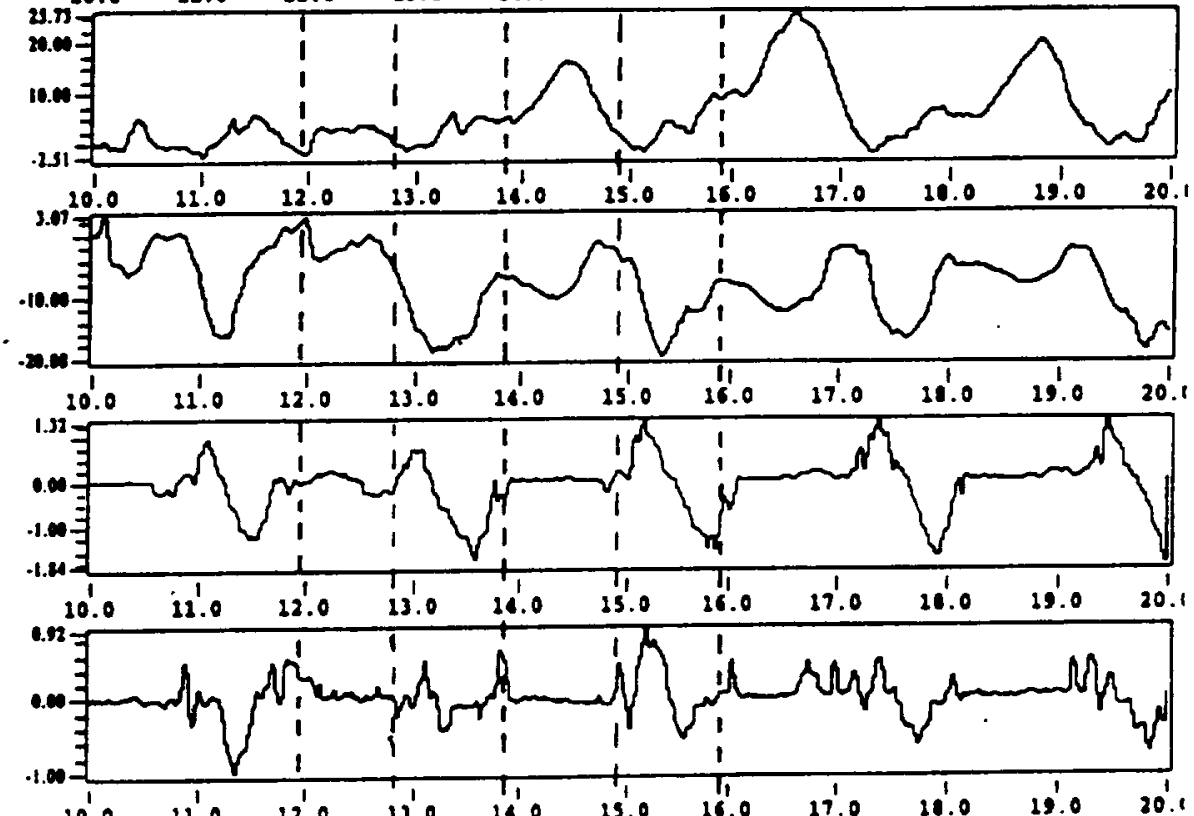

I Nous

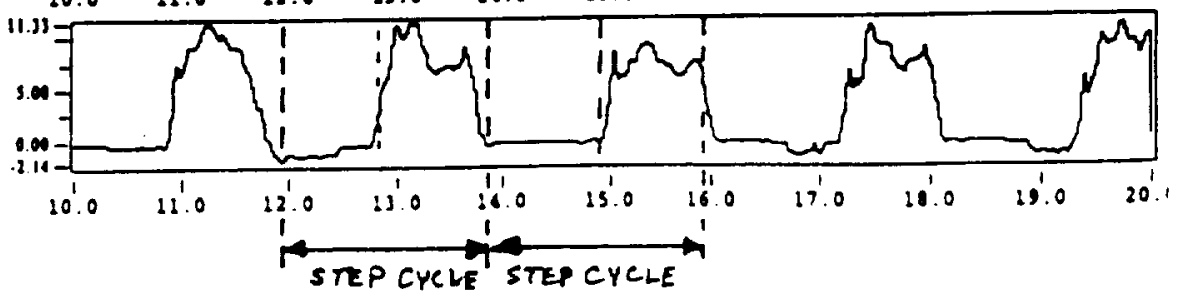

Figure 7. A I0-Sccond Recording Datat Sol During Tralining of An Acute SCI Paticnt 